\title{
A Performance Bound for Interpolation of MIMO-OFDM Channels
}

\author{
Michael Larsen and A. Lee Swindlehurst \\ School of Electrical and Computer Engineering \\ Brigham Young University \\ Provo, Utah 84602 \\ Email: mikedl@byu.edu, swindle@ee.byu.edu
}

\author{
Thomas Svantesson \\ ArrayComm, Inc. \\ San Jose, California 95131 \\ Email: tomaso@arraycomm.com
}

\begin{abstract}
The performance of MIMO-OFDM communication systems depends upon accurate channel estimation across frequency bands much wider than the coherence bandwidth of the channel. Obtaining channel estimates at all necessary frequencies typically involves interpolation of channel estimates obtained using pilots at a few distinct frequencies. This paper derives a performance bound for channel estimates obtained via frequency interpolation for slowly-fading frequency-selective channels. Numerical simulations of the bound indicate that normalized interpolation performance improves significantly as the number of antennas increases, suggesting that fewer pilots are needed as antennas are added to a MIMO-OFDM system.
\end{abstract}

\section{INTRODUCTION}

Wireless broadband multiple-input multiple-output (MIMO) communication systems, such as WiMax and other MIMO orthogonal frequency-division multiplexing (MIMO-OFDM) systems, require accurate channel estimates in time and in frequency. Typically this estimation is carried out by using pilot symbols to estimate the channel at select times and frequencies. In [1], it is shown that the optimal pilot sequences for OFDM are equipowered and equispaced over the frequency band. A similar result is shown in [2] for the MIMO-OFDM case. If the channel varies rapidly enough in frequency, interpolation or extrapolation may be necessary to obtain estimates at all of the frequencies of interest. The need for interpolation may also arise when the transmitter requires channel information and only limited feedback is available [3].

In order to quantify how well the channel may be estimated in frequency-selective channels through interpolation, we desire to find a lower bound on the interpolation performance for MIMO-OFDM systems. In [4], a performance bound is presented for temporal prediction and interpolation of narrowband fast-fading MIMO channels. In this work, we use a derivation similar to that of [4] to derive a performance bound for the frequency interpolation of wideband (frequency selective) slowly-fading MIMO channels. This lower-bound on the interpolation error is derived using a vector formulation of the Cramér-Rao bound and serves as a complement to the bound derived in [4].

This work was supported by the U.S. Army Research Office under the Multi-University Research Initiative (MURI) grant W911NF-04-1-0224, by the National Science Foundation under grant CCF-0313056, and by a National Science Foundation Graduate Research Fellowship.
The remainder of this paper is organized as follows. In Section II, we present the channel model which we will use as the foundation of the performance bound derivation. In Section III, we present the performance bound for the interpolation of frequency-selective, slowly-fading MIMO-OFDM channels. Numerical results for the bound are shown in Section IV. Finally, we present our conclusions in Section V.

\section{System ModeL}

The performance analysis is based on a ray-based MIMO channel model. This model is an extension of the lowpass single-input single-output (SISO) multipath fading model of [5] given by

$$
h(\tau ; t)=\sum_{l=1}^{L} \alpha_{l}(t) e^{-j \omega_{c} \tau_{l}(t)} \delta\left(\tau-\tau_{l}(t)\right)
$$

where $t$ is the time variable, $\tau$ is the delay variable, and $\omega_{c}$ is the carrier frequency. Each of the $L$ rays, or paths, has an associated scattering coefficient $\alpha_{l}(t)$ and path delay $\tau_{l}(t)$.

Since we are considering frequency-selective, slowly-fading channels, we remove the dependency on time to obtain

$$
h(\tau)=\sum_{l=1}^{L} \alpha_{l} e^{-j \omega_{c} \tau_{l}} \delta\left(\tau-\tau_{l}\right) .
$$

In the frequency domain, this model becomes

$$
h(\omega)=\sum_{l=1}^{L} \alpha_{l} e^{j\left(\omega_{c}-\omega\right) \tau_{l}}
$$

The model may be expanded to address MIMO channels with $M_{t}$ transmit antennas and $M_{r}$ receive antennas by introducing the spatial dimension to the model via transmit and receive array responses. The resulting model

$$
\mathbf{H}(\omega)=\sum_{l=1}^{L} \alpha_{l} \mathbf{a}_{r, l} \mathbf{a}_{t, l}^{T} e^{-j\left(\omega-\omega_{c}\right) \tau_{l}}
$$

where $\mathbf{a}_{t, l}$ and $\mathbf{a}_{r, l}$ are the transmit and receive array responses, respectively, is a wideband, slowly-fading MIMO model. It is valid for all types of antenna arrangements, but 
if uniform linear arrays (ULA) are used, the array response vectors have the structure

$$
\mathbf{a}_{\cdot, l}^{T}=\left[\begin{array}{lllll}
1 & e^{-j \Omega \cdot, l} & e^{-j 2 \Omega \cdot, l} & \cdots & e^{-j(M \cdot-1) \Omega_{\cdot, l}}
\end{array}\right],
$$

where $\Omega_{t, l}=k d_{t} \sin \phi_{t, l}, k$ is the wavenumber, $d_{t}$ is the antenna separation distance, and $\phi_{t, l}$ is the direction of departure. The parameter $\Omega_{r, l}$ is defined in a similar manner. Note that the array response vectors are functions of frequency through the wavenumber $k$. At the carrier frequencies and bandwidths of interest, this dependence on frequency is weak, so we will not explicitly represent it in our model. However, we will account for this frequency dependence later in the simulation examples.

The summation of (4) may be expressed in matrix-vector notation as

$$
\mathbf{H}(\omega)=\mathbf{A}_{r} \mathbf{X W}(\omega) \mathbf{A}_{t}^{T}
$$

where

$$
\begin{aligned}
\mathbf{A}_{r} & =\left[\mathbf{a}_{r, 1}, \ldots, \mathbf{a}_{r, L}\right] \\
\mathbf{A}_{t} & =\left[\mathbf{a}_{t, 1}, \ldots, \mathbf{a}_{t, L}\right] \\
\mathbf{X} & =\operatorname{diag}\left(\alpha_{1}, \ldots, \alpha_{L}\right) \\
\mathbf{W}(\omega) & =\operatorname{diag}\left(e^{j\left(\omega_{c}-\omega\right) \tau_{1}}, \ldots, e^{j\left(\omega_{c}-\omega\right) \tau_{L}}\right) .
\end{aligned}
$$

For the purpose of analysis, it is convenient to vectorize (6) to obtain

$$
\mathbf{h}(\omega)=\operatorname{vec}(\mathbf{H}(\omega))=\left(\mathbf{A}_{t} \otimes \mathbf{A}_{r} \mathbf{X}\right) \operatorname{vec}(\mathbf{W}(\omega))
$$

where $\operatorname{vec}(\cdot)$ is the vectorization operator and $\otimes$ indicates the Kronecker product.

We assume that we have $N_{M}$ channel estimates $\tilde{\mathbf{h}}\left(\omega_{n}\right)$ at various frequencies $\omega_{n}, n=1, \ldots, N_{M}$ to be used for interpolation. These estimates may be obtained through the use of training or pilot symbol transmission. We model these estimates as the true channel $\mathbf{h}\left(\omega_{n}\right)$ plus zero mean additive Gaussian noise $\mathbf{n}\left(\omega_{n}\right)$ so that

$$
\tilde{\mathbf{h}}\left(\omega_{n}\right)=\mathbf{h}\left(\omega_{n}\right)+\mathbf{n}\left(\omega_{n}\right) .
$$

The notation $\sim$ is used to indicate a channel estimate formed through training. The notation $\hat{\imath}$ will be used later to signify channel estimates obtained through interpolation. The noise distribution leads to the channel estimates being distributed as $\tilde{\mathbf{h}}\left(\omega_{n}\right) \sim \mathcal{C} \mathcal{N}\left(\mathbf{h}\left(\omega_{n}\right), \mathbf{C}\right)$. The noise is uncorrelated in space and frequency so that $\mathbf{C}=\sigma \mathbf{I}$. This is a good model when unitary training signals are used to estimate the channel, which is a reasonable assumption.

Stacking the $N_{M}$ estimates, we obtain the full model

$$
\begin{aligned}
& \tilde{\mathbf{h}}=\left[\begin{array}{llll}
\tilde{\mathbf{h}}\left(\omega_{1}\right)^{T} & \tilde{\mathbf{h}}\left(\omega_{2}\right)^{T} & \cdots & \tilde{\mathbf{h}}\left(\omega_{N_{M}}\right)^{T}
\end{array}\right]^{T} \\
& =\left[\begin{array}{c}
\left(\mathbf{A}_{t} \otimes \mathbf{A}_{r} \mathbf{X}\right) \operatorname{vec}\left(\mathbf{W}\left(\omega_{1}\right)\right) \\
\left(\mathbf{A}_{t} \otimes \mathbf{A}_{r} \mathbf{X}\right) \operatorname{vec}\left(\mathbf{W}\left(\omega_{2}\right)\right) \\
\vdots \\
\left(\mathbf{A}_{t} \otimes \mathbf{A}_{r} \mathbf{X}\right) \operatorname{vec}\left(\mathbf{W}\left(\omega_{L}\right)\right)
\end{array}\right]+\left[\begin{array}{c}
\mathbf{n}\left(\omega_{1}\right) \\
\mathbf{n}\left(\omega_{2}\right) \\
\vdots \\
\mathbf{n}\left(\omega_{N_{M}}\right)
\end{array}\right] \\
& =\left(\mathbf{I}_{N_{M}} \otimes\left(\mathbf{A}_{t} \otimes \mathbf{A}_{r} \mathbf{X}\right)\right) \mathcal{W}+\mathbf{n} \\
& =\mathbf{h}+\mathbf{n}
\end{aligned}
$$

where

$$
\mathcal{W}=\left[\begin{array}{c}
\operatorname{vec}\left(\mathbf{W}\left(\omega_{1}\right)\right) \\
\operatorname{vec}\left(\mathbf{W}\left(\omega_{2}\right)\right) \\
\vdots \\
\operatorname{vec}\left(\mathbf{W}\left(\omega_{N_{M}}\right)\right)
\end{array}\right]
$$

This model is parameterized by the $L$-length vectors $\alpha, \tau$, $\boldsymbol{\Omega}_{t}$, and $\boldsymbol{\Omega}_{r}$ and the scalar $\sigma$, where $\alpha$ is defined as $\alpha^{T}=$ $\left[\alpha_{1}, \ldots, \alpha_{L}\right]^{T}$ and the other vectors are defined similarly. To simplify analysis, we collect all of these parameters into the single $(5 L+1)$ real-valued vector $\theta=\left[\sigma, \Re[\alpha]^{T}, \Im[\alpha]^{T}, \tau^{T}\right.$, $\left.\boldsymbol{\Omega}_{\mathbf{t}}{ }^{T}, \boldsymbol{\Omega}_{\mathbf{r}}{ }^{T}\right]^{T}$, where $\Re[\cdot]$ and $\Im[\cdot]$ denote the real and imaginary parts of $(\cdot)$, respectively.

\section{LOWER BOUND ON INTERPOLATION ERROR}

We assume $N_{M}$ frequency-spaced channel estimates from (12) are available for use in interpolating the MIMO channel in frequency. In order to derive a lower bound for the interpolation error, we assume the following interpolation scheme: first, the $N_{M}$ channel estimates are found; next, the channel parameters $\theta$ are estimated using the available channel estimates; finally, interpolation is carried out using the model of (4) and the parameter estimates $\hat{\theta}$. We have chosen the ray-based channel model and this interpolation method with the purpose of finding the best possible performance of any frequency interpolator. Note that the estimation of the $(5 L+1)$ parameter values of $\theta$ may be extremely difficult for even moderate values of $L$. Thus, while this method of interpolation is convenient for our analysis and provides a useful lower bound, we do not mean to suggest this method as a preferred algorithm for interpolation. In practice, the use of other interpolation models and algorithms may be more appropriate for particular implementations.

The estimation error using the aforementioned interpolation scheme at any frequency $\omega$ is

$$
\begin{aligned}
\mathbf{e}(\omega) & =\hat{\mathbf{h}}(\omega, \hat{\theta})-\mathbf{h}(\omega, \theta) \\
& =\sum_{l=1}^{L}\left(\hat{\mathbf{a}}_{t, l} \otimes \hat{\mathbf{a}}_{r, l} \hat{\alpha}_{l} e^{j \Delta \omega \hat{\tau}_{l}}-\mathbf{a}_{t, l} \otimes \mathbf{a}_{r, l} \alpha_{l} e^{j \Delta \omega \tau_{l}}\right)
\end{aligned}
$$

where $\Delta \omega=\omega_{c}-\omega$. It is clear from (15) that the estimate error is a nonlinear function of the parameters, and we cannot find the a minimizing solution of $\mathbf{e}(\omega)$ directly. Instead, we use the Cramér-Rao bound (CRB) to find the lower bound for the error covariance matrix of any unbiased estimator. This bound may be written using a vector formulation of the CRB for functions of parameters [6] as

$$
E\left[(\hat{\mathbf{h}}(\omega)-\mathbf{h}(\omega))(\hat{\mathbf{h}}(\omega)-\mathbf{h}(\omega))^{H}\right] \geq \mathbf{H}^{\prime} \mathbf{B} \mathbf{H}^{\prime H}
$$

where the matrix inequality $\mathbf{A} \geq \mathbf{B}$ means that $\mathbf{A}-\mathbf{B}$ is positive semi-definite. The matrix $\mathbf{H}^{\prime}$ is the $\left(M_{t} M_{r}\right) \times(5 L+1)$ Jacobian matrix

$$
\mathbf{H}^{\prime}=\left[\begin{array}{llll}
\frac{\partial \mathbf{h}(\omega)}{\partial \theta_{1}} & \frac{\partial \mathbf{h}(\omega)}{\partial \theta_{2}} & \cdots & \frac{\partial \mathbf{h}(\omega)}{\partial \theta_{5 L+1}}
\end{array}\right] .
$$


The matrix $\mathbf{B}$ defines the CRB for the parameters $\theta$, and it can be expressed using Bangs formula [7] as

$$
\begin{aligned}
{\left[\mathbf{B}^{-1}\right]_{i j}=[\mathbf{J}(\theta)]_{i j}=} & \operatorname{Tr}\left[\mathbf{C}^{-1} \frac{\partial \mathbf{C}}{\partial \theta_{i}} \mathbf{C}^{-1} \frac{\partial \mathbf{C}}{\partial \theta_{j}}\right] \\
& +2 \Re\left[\frac{\partial \mathbf{h}^{H}}{\partial \theta_{\mathbf{i}}} \mathbf{C}^{-1} \frac{\partial \mathbf{h}}{\partial \theta_{j}}\right]
\end{aligned}
$$

where $\mathbf{J}(\theta)$ is the Fisher matrix. Using (17) and (18), the CRB matrix of (16) may be calculated in a straightforward manner. Readers interested in the derivation may refer to the closelyrelated derivation in the appendix of [4] for details.

Using (18), the Fisher matrix is found to be

$\mathbf{J}(\theta)=\left[\begin{array}{cccccc}\frac{M_{t} M_{r} N_{M}}{\sigma} & \mathbf{0}^{T} & \mathbf{0}^{T} & \mathbf{0}^{T} & \mathbf{0}^{T} & \mathbf{0}^{T} \\ \mathbf{0} & \Re\left[\mathbf{P}_{1}\right] & -\Im\left[\mathbf{P}_{1}\right] & \Re\left[\mathbf{P}_{2}\right] & \Re\left[\mathbf{P}_{3}\right] & \Re\left[\mathbf{P}_{4}\right] \\ \mathbf{0} & \Im\left[\mathbf{P}_{1}\right] & \Re\left[\mathbf{P}_{1}\right] & \Im\left[\mathbf{P}_{2}\right] & \Im\left[\mathbf{P}_{3}\right] & \Im\left[\mathbf{P}_{4}\right] \\ \mathbf{0} & \Re\left[\mathbf{P}_{2}^{T}\right] & \Im\left[\mathbf{P}_{2}^{T}\right] & \Re\left[\mathbf{P}_{5}\right] & \Re\left[\mathbf{P}_{6}\right] & \Re\left[\mathbf{P}_{7}\right] \\ \mathbf{0} & \Re\left[\mathbf{P}_{3}^{T}\right] & \Im\left[\mathbf{P}_{3}^{T}\right] & \Re\left[\mathbf{P}_{6}^{T}\right] & \Re\left[\mathbf{P}_{8}\right] & \Re\left[\mathbf{P}_{9}\right] \\ \mathbf{0} & \Re\left[\mathbf{P}_{4}^{T}\right] & \Im\left[\mathbf{P}_{4}^{T}\right] & \Re\left[\mathbf{P}_{7}^{T}\right] & \Re\left[\mathbf{P}_{9}^{T}\right] & \Re\left[\mathbf{P}_{10}\right] \\ \end{array}\right.$

The submatrices $\mathbf{P}_{1}-\mathbf{P}_{10}$ are given by

$$
\begin{aligned}
& \mathbf{P}_{1}=\frac{2}{\sigma} \breve{\mathbf{W}}^{H}\left(\mathbf{I}_{N_{M}} \otimes \mathbf{A}_{t}^{H} \mathbf{A}_{t} \odot \mathbf{A}_{r}^{H} \mathbf{A}_{r}\right) \breve{\mathbf{W}} \\
& \mathbf{P}_{2}=\frac{2}{\sigma} \breve{\mathbf{W}}^{H}\left(\mathbf{I}_{N_{M}} \otimes \mathbf{A}_{t}^{H} \mathbf{A}_{t} \odot \mathbf{A}_{r}^{H} \mathbf{A}_{r} \mathbf{X}\right) \breve{\mathbf{D}}_{\tau} \\
& \mathbf{P}_{3}=\frac{2}{\sigma} \breve{\mathbf{W}}^{H}\left(\mathbf{I}_{N_{M}} \otimes \mathbf{A}_{t}^{H} \mathbf{D}_{t} \odot \mathbf{A}_{r}^{H} \mathbf{A}_{r} \mathbf{X}\right) \breve{\mathbf{W}} \\
& \mathbf{P}_{4}=\frac{2}{\sigma} \breve{\mathbf{W}}^{H}\left(\mathbf{I}_{N_{M}} \otimes \mathbf{A}_{t}^{H} \mathbf{A}_{t} \odot \mathbf{A}_{r}^{H} \mathbf{D}_{r} \mathbf{X}\right) \breve{\mathbf{W}} \\
& \mathbf{P}_{5}=\frac{2}{\sigma} \breve{\mathbf{D}}_{\tau}^{H}\left(\mathbf{I}_{N_{M}} \otimes \mathbf{A}_{t}^{H} \mathbf{A}_{t} \odot \mathbf{X}^{H} \mathbf{A}_{r}^{H} \mathbf{A}_{r} \mathbf{X}\right) \breve{\mathbf{D}}_{\tau} \\
& \mathbf{P}_{6}=\frac{2}{\sigma} \breve{\mathbf{D}}_{\tau}^{H}\left(\mathbf{I}_{N_{M}} \otimes \mathbf{A}_{t}^{H} \mathbf{D}_{t} \odot \mathbf{X}^{H} \mathbf{A}_{r}^{H} \mathbf{A}_{r} \mathbf{X}\right) \breve{\mathbf{W}} \\
& \mathbf{P}_{7}=\frac{2}{\sigma} \breve{\mathbf{D}}_{\tau}^{H}\left(\mathbf{I}_{N_{M}} \otimes \mathbf{A}_{t}^{H} \mathbf{A}_{t} \odot \mathbf{X}^{H} \mathbf{A}_{r}^{H} \mathbf{D}_{r} \mathbf{X}\right) \breve{\mathbf{W}} \\
& \mathbf{P}_{8}=\frac{2}{\sigma} \breve{\mathbf{W}}^{H}\left(\mathbf{I}_{N_{M}} \otimes \mathbf{D}_{t}^{H} \mathbf{D}_{t} \odot \mathbf{X}^{H} \mathbf{A}_{r}^{H} \mathbf{A}_{r} \mathbf{X}\right) \breve{\mathbf{W}} \\
& \mathbf{P}_{9}=\frac{2}{\sigma} \breve{\mathbf{W}}^{H}\left(\mathbf{I}_{N_{M}} \otimes \mathbf{D}_{t}^{H} \mathbf{A}_{t} \odot \mathbf{X}^{H} \mathbf{A}_{r}^{H} \mathbf{D}_{r} \mathbf{X}\right) \breve{\mathbf{W}} \\
& \mathbf{P}_{10}=\frac{2}{\sigma} \breve{\mathbf{W}}^{H}\left(\mathbf{I}_{N_{M}} \otimes \mathbf{A}_{t}^{H} \mathbf{A}_{t} \odot \mathbf{X}^{H} \mathbf{D}_{r}^{H} \mathbf{D}_{r} \mathbf{X}\right) \breve{\mathbf{W}}
\end{aligned}
$$

where $\odot$ is the element-wise matrix product (Hadamard product) and

$$
\begin{aligned}
\breve{\mathbf{W}}= & {\left[\mathbf{W}\left(\omega_{1}\right)^{T}, \ldots, \mathbf{W}\left(\omega_{N_{M}}\right)^{T}\right]^{T} } \\
\breve{\mathbf{D}}_{\tau}= & {\left[\mathbf{D}_{\tau}\left(\omega_{1}\right)^{T}, \ldots, \mathbf{D}_{\tau}\left(\omega_{N_{M}}\right)^{T}\right]^{T} } \\
\mathbf{D}_{\tau}(\omega)= & \operatorname{diag}\left(\left(\omega_{c}-\omega\right) e^{j\left(\omega_{c}-\omega\right) \tau_{1}}\right. \\
& \left.\ldots,\left(\omega_{c}-\omega\right) e^{j\left(\omega_{c}-\omega\right) \tau_{L}}\right) \\
\mathbf{D}_{t}= & {\left[\frac{\partial\left[\mathbf{A}_{t}\right]_{:, 1}}{\partial \Omega_{t, 1}}, \ldots, \frac{\partial\left[\mathbf{A}_{t}\right]_{:, L}}{\partial \Omega_{t, L}}\right] } \\
\mathbf{D}_{r}= & {\left[\frac{\partial\left[\mathbf{A}_{r}\right]_{:, 1}}{\partial \Omega_{r, 1}}, \ldots, \frac{\partial\left[\mathbf{A}_{r}\right]_{: L}}{\partial \Omega_{r, L}}\right] . }
\end{aligned}
$$

The sum of the variances of the interpolation errors for the channel matrix coefficients may be bounded as

$$
E\left[\|\mathbf{e}(\omega)\|_{F}^{2}\right] \geq \operatorname{Tr}\left[\mathbf{H}^{\prime} \mathbf{B} \mathbf{H}^{\prime H}\right]=\varepsilon_{0}(\omega)
$$

where $\|\cdot\|_{F}$ denotes the Frobenius norm and the argument of the trace is the CRB matrix given by (16).

\section{NUMERICAL Simulations}

In the previous section, we derived a performance bound for the frequency interpolation of wideband, slowly-fading MIMO channels. In this section, we numerically evaluate the performance bounds for a few specific scenarios. To quantify the performance, we use the root mean square error (RMSE) measure

$$
\sqrt{\frac{E\left[\left\|\mathbf{e}\left(\omega_{c}+\omega_{Q}\right)\right\|_{F}^{2}\right]}{E\left[\left\|\mathbf{H}\left(\omega_{c}+\omega_{Q}\right)\right\|_{F}^{2}\right]}} \geq \sqrt{\frac{\varepsilon_{0}\left(\omega_{c}+\omega_{Q}\right)}{E\left[\left\|\mathbf{H}\left(\omega_{c}+\omega_{Q}\right)\right\|_{F}^{2}\right]}}=\varepsilon(Q)
$$

where $\omega_{c}$ denotes the signal carrier frequency and $\omega_{Q}$ represents the frequency relate to $\omega_{c}$ at which the channel is interpolated. From our discussion of (35), we recognize that this error measure represents a bound on the average error of all of the channel matrix elements. This allows us to fairly compare the results of simulations for SISO and MIMO channels.

\section{A. Simulation Setup}

To reduce the complexity of our simulations, we make several simplifying assumptions concerning our channel parameters. The assumptions are used in the construction of simulated channels for which the bound will be evaluated. First, we assume that the different parameters

$$
\theta=\left[\sigma, \Re[\alpha]^{T}, \Im[\alpha]^{T}, \tau^{T}, \boldsymbol{\Omega}_{\mathbf{t}}^{T}, \boldsymbol{\Omega}_{\mathbf{r}}^{T}\right]^{T}
$$

are independent. This ignores the tendency for larger magnitude scattering parameters to be associated with lower delays. However, we believe this assumption is reasonable for our purposes. We also assume the scattering parameters $\alpha$ are zero-mean unit-variance complex Gaussian distributed, i.e., $\alpha \sim \mathcal{C N}(0,1)$. A uniform distribution is assumed for the path delays, $\tau_{l}$, so that $\tau_{l} \sim U[40 n s, 400 n s]$. The antenna arrays are assumed to be ULAs at both the transmitter and receiver. The angles of arrival and departure for the ULAs for path $l$ are defined as $\Omega_{r, l}=k d_{r} \sin \phi_{r, l}$ and $\Omega_{r, l}=k d_{r} \sin \phi_{r, l}$, respectively, with antenna spacings $d_{r}=d_{t}=\lambda / 2$ at $\omega_{c}=2.4 \mathrm{GHz}$ and angles distributed uniformly as $\phi_{r}, \phi_{t} \sim U[0,2 \pi)$.

For these parameter distributions, the average channel power simplifies to $E\left[\|\mathbf{H}(\omega)\|_{F}^{2}\right]=L M_{t} M_{r}$. Thus, the normalized interpolation error bound of (35) becomes

$$
\epsilon(\omega)=\sqrt{\frac{\operatorname{Tr}\left[\mathbf{H}^{\prime} \mathbf{B H}^{\prime H}\right]}{L M_{t} M_{r}}} .
$$

The SNR for our simulations is defined as

$$
\mathrm{SNR}=\frac{E\left[\|\mathbf{h}(\omega)\|^{2}\right]}{E\left[\|\mathbf{n}(\omega)\|^{2}\right]}=\frac{L}{\sigma} .
$$

For our simulations, we keep the noise power fixed at $-20 \mathrm{~dB}$. Thus, the SNR in our simulations is not fixed, but is dependent on the number of paths. 


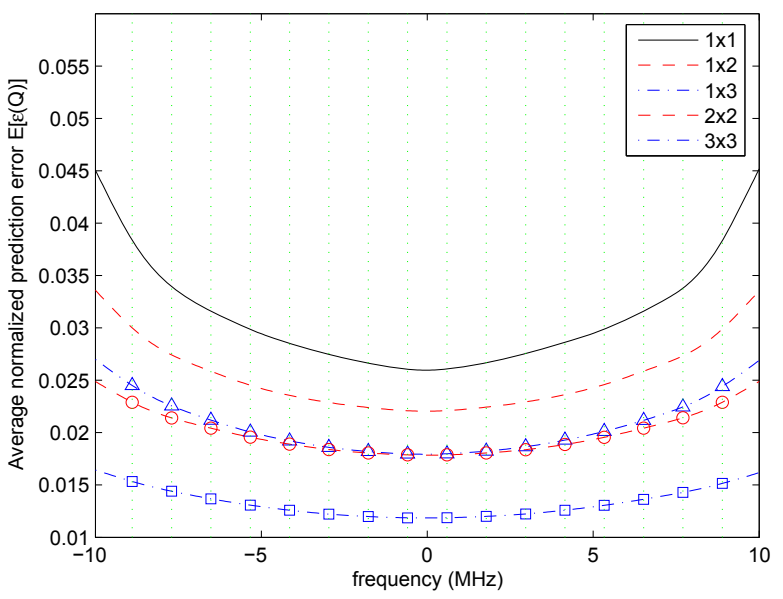

Fig. 1. Average normalized interpolation error for 2-path SISO and MIMO systems versus frequency.

\section{B. Two Path Example}

First, we present numerical results for a two path scenario. Using the parameter distribution assumptions discussed previously, we have randomly created 100 channel realizations using the channel model of (4). We assume that we have $N_{M}=16$ channel estimates evenly distributed over a bandwidth of $20 \mathrm{MHz}$. For each channel realization, the normalized interpolation error bound of (38) is found over the bandwidth. Figure 1 is a plot of the normalized error bound averaged over the channel realizations. The vertical dotted lines on the plot indicate the locations of the $N_{M}$ channel estimates. The results indicate that the ability to interpolate increases as the number of transmit and receive antennas increases. A intuitive explanation for this result is that the additional antennas in a MIMO system reveal more information about the channel structure, allowing for better interpolation performance when compared to a SISO system. A mathematical explanation is that the SISO and MIMO systems each require the same number of parameters $\theta$ to be estimated. The MIMO systems, however, have more data points per channel sample to use in carrying out the parameter estimation. The shape of the curves in this figure, higher on the outsides with the smallest error occurring in the middle, is not surprising since channel samples on both sides of the frequency in question contribute to a lower interpolation error. However, this shape does suggest that in this particular scenario, uniform spacing of the channel estimates, i.e., the location of the pilot symbols, favors the frequencies at the center of the bandwidth. Other spacing schemes, with more channel estimates towards the outside of the bandwidth, may be able to flatten these curves over more of the bandwidth.

\section{Eight Path Example}

We now consider a channel with $L=8$ multipath components. The results in [8] for outdoor measurements in Austin, TX, suggest that channels may be better modeled using 3-8

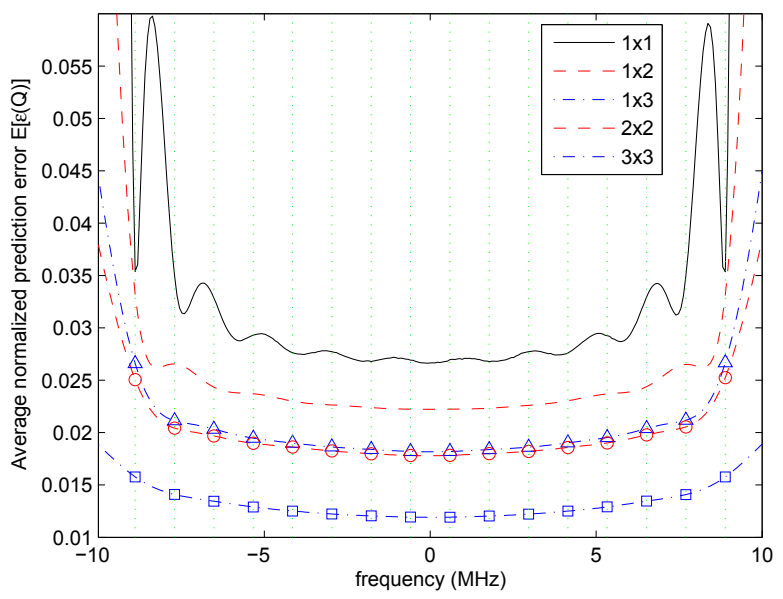

Fig. 2. Average normalized interpolation error for 8-path SISO and MIMO systems versus frequency.

multipath components. Hence, this example represents a more realistic scenario. We use the same simulation setup for the eight path channel as for the two path channel. Note that as we increase the number of paths, an identifiability problem will occasionally arise when two paths have similar parameters, resulting in an ill-conditioned CRB matrix. This issue is particularly pronounced in the SISO case and becomes less of a problem as the number of antennas increases. We address this problem by removing the 15 channel realizations with the poorest conditioned $\mathrm{CRB}$ matrices from consideration. Figure 2 shows the average normalized error bound versus frequency for the $L=8$ case. As in the two path example, the results of the eight-path scenario indicate that the ability to interpolate increases as the number of transmit and receive antennas increases. Note that the curves for the $L=8$ case are more uniform across frequency that in the $L=2$ case. Thus, for channels with a larger number of multipath components, uniform spacing of the $N_{M}$ channel estimates allows for good interpolation performance over most of the bandwidth under consideration. The oscillations present in the SISO curve indicate that, in this example, not enough channel estimates are available to effectively interpolate between channel estimates. The channel estimates are simply too far apart for the SISO interpolator to perform well.

We further investigate this channel estimate spacing issue in Fig. 3. This figure is a plot of the interpolation error bound, averaged over channel realizations and frequency, versus the spacing between the $N_{M}$ channel estimates in frequency. The number of available channel estimates is fixed at $N_{M}=16$ and the spacing between these estimates increases as we move to the right of the plot. This implies that the bandwidth increases to the right, as well. Also included on the plot is an estimate of the average coherence bandwidth (CBW) of the channel realizations. We estimate the CBW for each channel realization by the inverse of the maximum delay spread for that realization. The average CBW plotted is the 


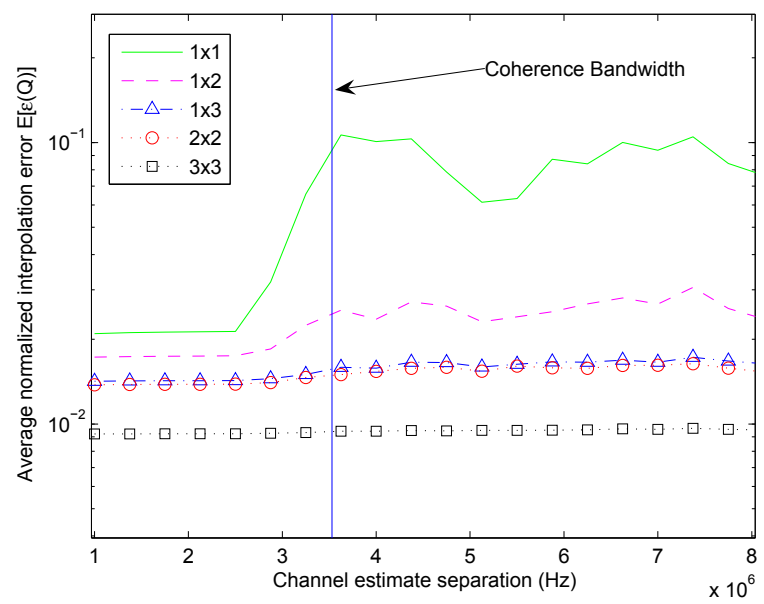

Fig. 3. Average normalized interpolation error for SISO and MIMO systems versus the frequency separation of the $N_{M}$ training-based channel estimates.

mean of the CBW for all the realizations. Other methods for calculating in the delay spread, such as those given in [9], are available. However, the method we use to calculate the delay spread seems the best matched with our channel model and simplifying parameter assumptions. In Fig. 3, we note that as the frequency separation approaches the $\mathrm{CBW}$ of the channel, a rapid decrease in interpolation performance is observed in the SISO case. A similar decrease is observed in the $1 \times 2,1 \times 3$ and $2 \times 2$ MIMO cases, as well. However, the decrease in performance begins later for the MIMO channels than for the SISO channels. For the $3 \times 3$ MIMO channel, very little decrease in performance is observed, even after the the channel estimates are spaced farther apart than the CBW. This suggests that for MIMO channels, the channel estimates generated through training may be spaced father apart in frequency than for SISO channels, and in some cases, farther apart than the CBW.

\section{Limitations of the Performance Bound}

As the results previously presented demonstrate, the performance bound for the interpolation of MIMO-OFDM channels provides useful insights into the benefits of MIMO systems in terms of interpolation error performance. However, some caution is advisable when interpreting the results given by the bound due to some inherent limitations of the bound. In the formulation of the CRB of (16), it was implicitly assumed that the true channel is perfectly modeled by (4) and that interpolation errors are a result of noisy channel estimates. Since (4) generally will not perfectly model the channel, there will be unaccounted for modeling errors when applying this bound to true channels. Therefore, the bound will not be fully achievable, and the tightness of the bound will depend in part on the amount of modeling error present. Note that we expect the impact of modeling errors to be most pronounced when the model is used to extrapolate the channel.

It should also be recognized that the channel given by the model of (4) is a sum of sinusoids, and is, therefore, periodic in frequency, though the period may be large for large $L$. Thus, care should be exercised when applying the bound over a large range of frequencies to ensure that channel is still behaving "realistically enough" for the results to be meaningful. Also, channel statistics such as the CBW should be used with caution since the modeled channels may exhibit cyclic correlation trends. For example, results for the $L=8$ scenario do not appear to be valid much beyond the bandwidths used in Fig. 3 . However, bandwidths currently considered for MIMO-OFDM systems are typically well within this applicable range.

\section{CONCLUSION}

In this work, we have derived a lower bound for the frequency interpolation error in wideband, slowly-fading MIMO channel estimation schemes. This bound particularly addresses the situation in MIMO-OFDM systems in which a few channel estimates found through training must be interpolated to all frequencies of interest. The bound was found by finding the CRB for the interpolation using a MIMO ray-based channel model. Numerical analysis of the bound indicates that the interpolation performance is significantly improved when multiple antennas are used at the transmitter and receiver. We attribute this performance increase to the additional channel structure revealed in the MIMO case relative to the number of parameters estimates required for channel interpolation. The bound is most applicable when the true channel is well described by the ray-based model.

\section{REFERENCES}

[1] S. Ohno and G. B. Giannakis, "Optimal training and redundant precodingfor block transmissions with application to wireless OFDM," IEEE Trans. Commun., vol. 50, pp. 2113-2123, Dec. 2002.

[2] I. Barhumi, G. Leus, and M. Moonen, "Optimal training design for MIMO OFDM systems in mobile wireless channels," IEEE Trans. Signal Processing, vol. 51, pp. 1615-1624, June 2003.

[3] J. Choi and R. W. Heath, "Interpolation based transmit beamforming for MIMO-OFDM with limited feedback," IEEE Trans. Signal Processing, vol. 53, pp. 4125-4135, Nov. 2005.

[4] T. Svantesson and A. L. Swindlehurst, "A performance bound for the prediction of MIMO channels," IEEE Trans. Signal Processing, vol. 54, pp. 520-529, Feb. 2006.

[5] J. G. Proakis, Digital Communications, forth ed. New York, NY: McGraw-Hill, 2001, ch. 14.

[6] S. M. Kay, Fundamentals of Statistical Signal Processing. Englewood Cliffs, NJ: Prentice-Hall, 1993.

[7] W. J. Bangs, "Array processing with generalized beamformers," Ph.D. dissertation, Yale University, New Haven, CT, 1971.

[8] A. Arrendondo, K. Dandekar, and G. Xu, "Vector channel modeling and prediction for the improvement of downlink received power," IEEE Trans. Commun., vol. 50, pp. 1121-1129, July 2002.

[9] T. S. Rappaport, Wireless Communications: Principles and Practice. Upper Saddle River, NJ: Prentice Hall, 1996, ch. 4. 